\title{
Surgical treatment of rhinophyma using an ordinary bur
}

\author{
Jae Kyoung Kang ${ }^{1}$, \\ Jae Seong Lee ${ }^{2}$, \\ Jae Hyuck Choi ${ }^{3}$, \\ Myoung Soo Shin ${ }^{1}$, \\ Byung Min Yun ${ }^{1}$ \\ ${ }^{1}$ Department of Plastic and \\ Reconstructive Surgery, Jeju National \\ University School of Medicine, Jeju; \\ ${ }^{2}$ Department of Plastic and \\ Reconstructive Surgery, Jeju National \\ University Hospital, Jeju; ${ }^{3}$ Department \\ of General Surgery, Jeju National \\ University School of Medicine, Jeju, \\ Korea
}

Rhinophyma is a painless benign tumor of the skin of the nose. It is the most severe form of rosacea. Rhinophyma is particularly rare among Asians. It can be treated with surgical resection using various methods, including scalpel excision, dermabrasion, cryosurgery, argon laser, carbon dioxide laser, and electrocautery. However, the gold standard treatment of rhinophyma remains unknown. In the present case, we debulked a giant rhinophyma with a scalpel and contoured it using a bur. Along with a relevant literature review, we present a case of rhinophyma in an Asian patient who was treated at no additional cost using a bur widely used in general plastic surgery.

Keywords: Rhinophyma / Rosacea / Surgical instrument

\section{INTRODUCTION}

Rhinophyma is a rare and painless benign tumor of the skin of the nose characterized by hyperplasia of the sebaceous glands, connective tissue, and blood vessels [1]. Rhinophyma is generally confined to the lower nose and does not involve the bone or cartilage framework. However, giant rhinophymas distort the nasal aesthetics, and severe cases may cause airway obstruction, thereby compromising the patient's quality of life and emotional state [2]. There is no gold standard treatment for Rhinophyma; however, surgical treatments, such as scalpel excision, dermabrasion, cryosurgery, argon laser, carbon dioxide laser, and electrocautery, have been used to treat rhinophyma, and different treatments are applied depending on the patient's condition [3]. A dermabrader has the advantages of low cost

\footnotetext{
Correspondence: Byung Min Yun

Department of Plastic and Reconstructive Surgery, Jeju National University School of Medicine, 102 Jejudaehang-ro, Jeju 54987, Korea

E-mail: almostfree@hanmail.net

This work was supported by the 2020 education, research and student guidance grant funded by Jeju National University.

Received May 19, 2020 / Revised July 17, 2020 / Accepted August 14, 2020
}

and precise contouring around the nose. However, not many hospitals are equipped with this device nowadays, as other techniques have been developed for treating scarring, wrinkles, and pockmarks. However, drills and burs, which function under similar principles, are widely used in plastic surgery, and are commonly available in hospitals. In this paper, we present a rare case of rhinophyma in an Asian patient who was treated at no additional cost using a bur widely used in plastic surgery.

\section{IDEA}

The patient was a 72-year-old man with a 10-year history of telangiectasia and erythema of the nose and a 2-year history of induration, prominence, and thickening of the nasal tissues. Although there was no pain, the patient presented to our outpatient clinic because the compressed nares caused difficulty in breathing, and it was aesthetically unpleasant. The lesion affected the nasal bridge and had spread to the bilateral alar lobules (Fig. 1). Results of a biopsy performed at the outpatient clinic suggested rhinophyma; based on this, the patient was scheduled for surgical intervention. 

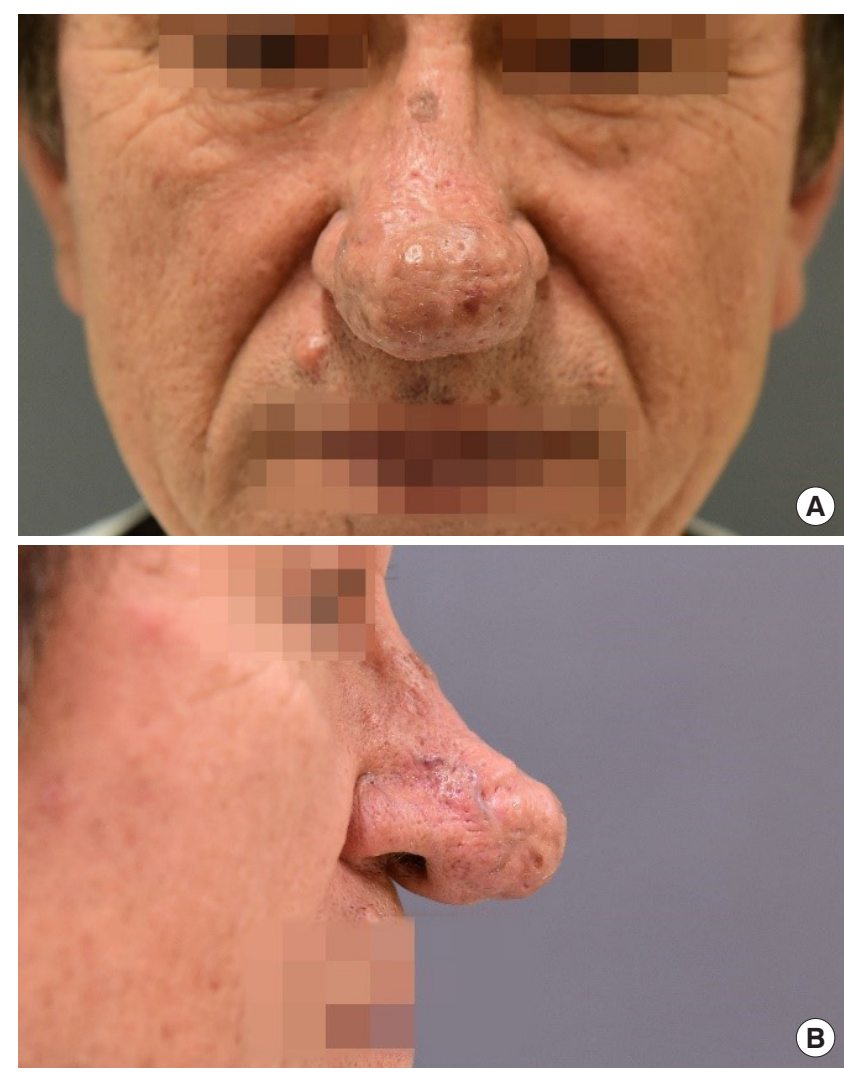

Fig. 1. Preoperative state frontal view (A) and lateral view (B).

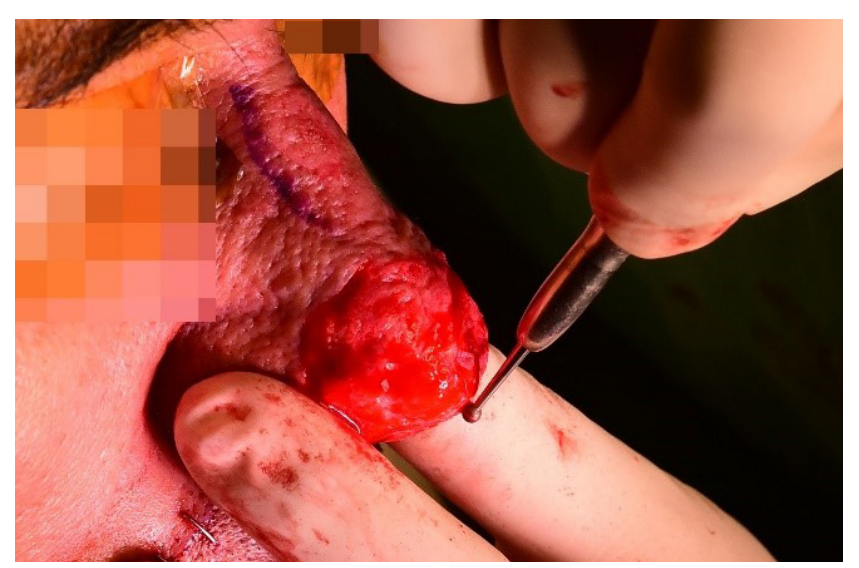

Fig. 2. Bone drill with a diamond bur to contour nasal tip.

The surgery was performed under general anesthesia. The resection areas were designed with consideration of the nasal aesthetic unit. A mixture of $150 \mathrm{U}$ hyaluronidase in $30 \mathrm{~mL}$ of 1:100,000 lidocaine/epinephrine was infiltrated. Then, the tumor was debulked with a \#15 scalpel, followed by nasal tip contouring using a round diamond bur (Fig. 2). Contouring was performed at a bur rotation speed of 30,000 revolutions per minute (rpm). We preserved the pilosebaceous appendage for spontaneous re-epithelialization and took precautions to avoid
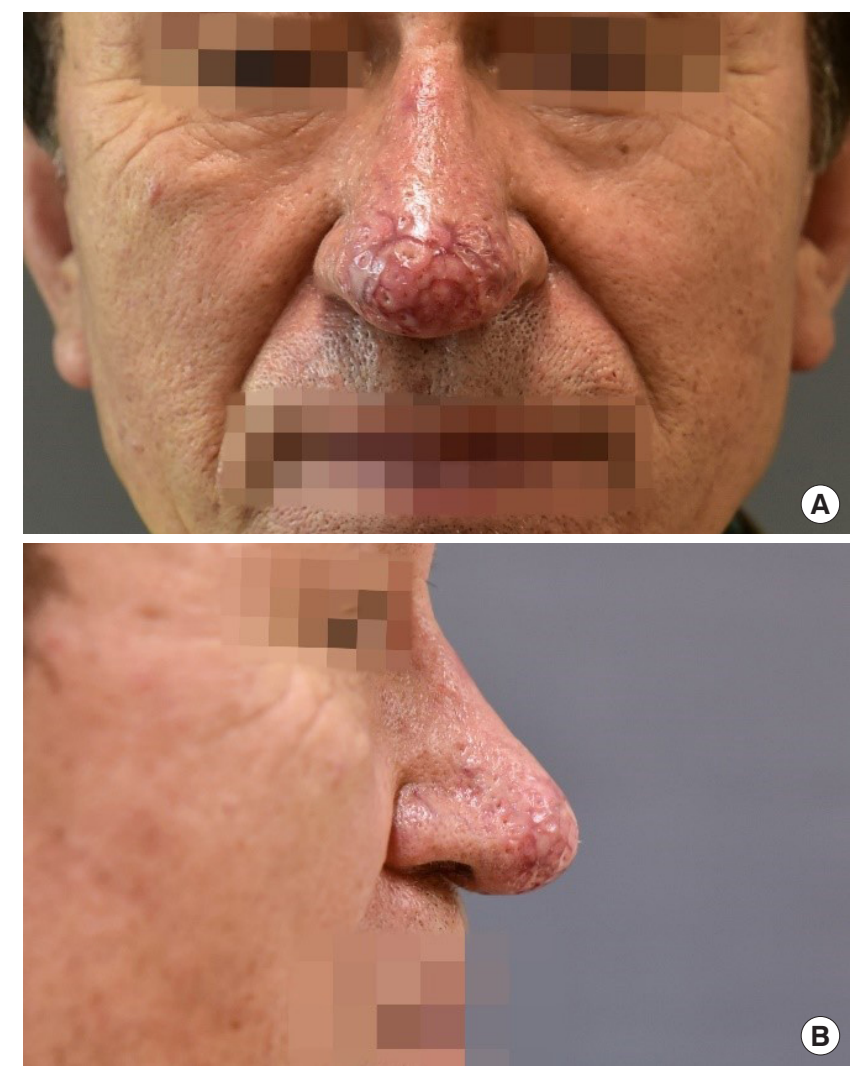

Fig. 3. Postoperative state (8 weeks) frontal view (A) and lateral view (B).

injury to the nasal cartilage. After achieving hemostasis using Bosmin gauze, a Vaseline wet dressing was applied. Postoperatively, the dressing was changed once every 2 days. The wound was re-epithelialized 2 weeks after surgery. During 8 weeks of observation after surgery, there were no side effects, such as inflammation, and the patient was very satisfied not only with the ease of the procedure but also with the aesthetic result (Fig. 3). The nasal height, measured from the vertical alar plane to the nasal tip, was reduced by $20 \%$ after surgery.

\section{DISCUSSION}

Rhinophyma is a benign tumor of unknown cause that grows very slowly, and its worldwide incidence and prevalence remain unknown. There are only about 20 cases reported among Asians [4]. Although the exact cause of rhinophyma remains elusive, excessive alcohol intake, vitamin deficiency, repeated infections, and sunlight exposure have been suspected as risk factors $[4,5]$. Rhinophyma is the most severe form of rosacea, developing in the course of prerosacea, vascular rosacea, and inflammatory rosacea, and it is clinically characterized by telangiectasia, thick skin, palpable nodules, enlarged pores, and foulsmelling sebum secretion in the lower nose [6]. This slow pro- 


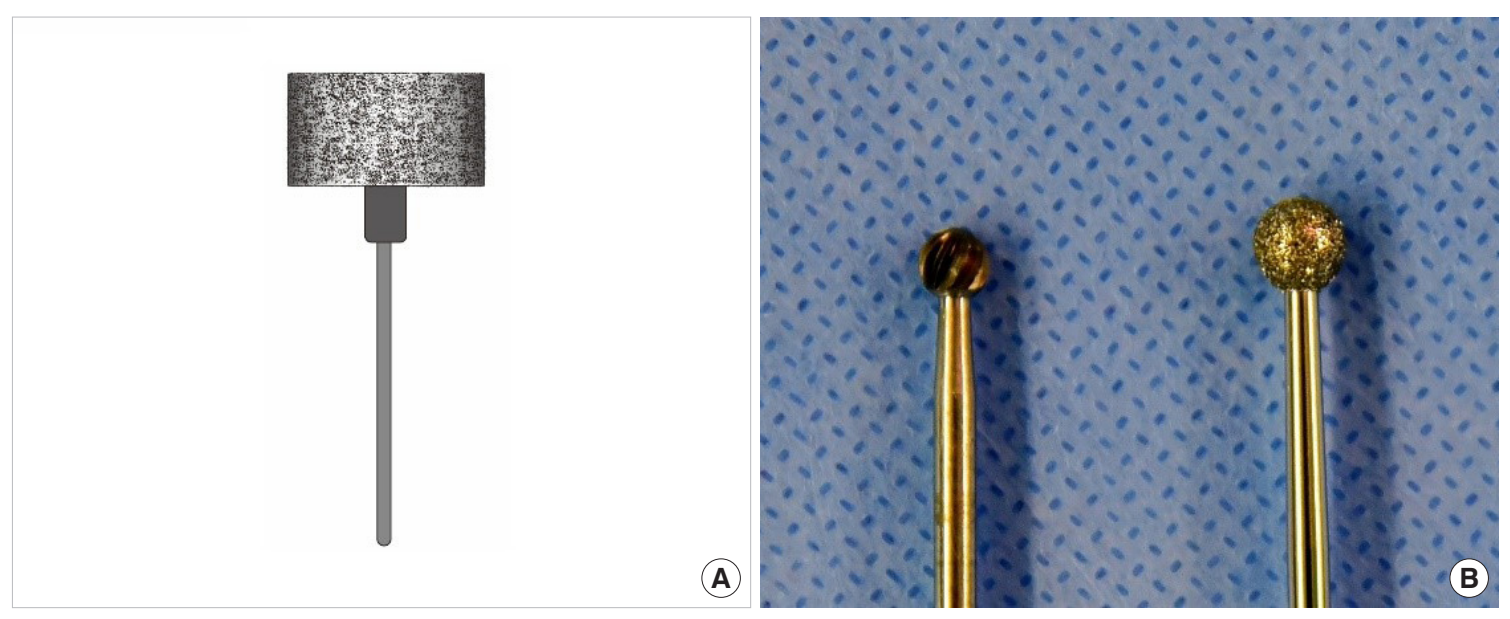

Fig. 4. Illustration of conventional diamond fraise for dermabrasion (A) and two kinds of ordinary bur (B); left: carbide bur; right: diamond bur.

gressive process begins in the teenage years or the twenties with rhinophyma generally diagnosed in the sixth decade of life $[4,6]$.

Although unusual, a malignant skin tumor may develop in the thickened tissue of rhinophyma; hence, a pathologic exam is essential. Basal cell carcinoma, squamous cell carcinoma, sebaceous carcinoma, and angiosarcoma have been reported, with basal cell carcinoma being the most common [7]. In this case, the biopsy results confirmed the absence of malignant tumors.

There have been reports that nonsurgical treatments, such as topical metronidazole, retinoids, low dose isotretinoin, and oral antibiotics, were effective in treating rhinophyma. However, these treatments cannot regress rhinophyma once it progresses. Giant rhinophymas are generally treated surgically with scalpel excision, dermabrasion, cryosurgery, argon or $\mathrm{CO}_{2}$ laser surgery, or electrocautery excision [3]. A variety of other surgical interventions, such as the ultrasonic scalpel, disposable razor, PlasmaBlade, Versajet, or a combination of existing techniques, have also been attempted. However, there is no gold standard technique, and several factors, including size and location of the lesion, hemostasis, scar formation, procedure duration, and cost associated with each instrument must be taken into consideration [3,8-11].

The advantages of scalpel excision and dermabrasion include low cost and precise contouring around the nasal aesthetic unit. In this case, we first performed mass debulking with a scalpel. In contrast to existing techniques, we used a bone drill and diamond bur, which are used in bone surgeries, for contouring. A dermabrader was used by attaching a handle and diamond fraise or wire brush to a power source. A diamond fraise (Fig. 4) has recently gained popularity owing to its ability to produce even depth easily [12]. A dermabrader can operate at 10,000$85,000 \mathrm{rpm}$ and is more effective at a higher speed $[12,13]$.
While dermabraders have been used to treat scars, wrinkles, and pockmarks in the past, with the drastic decrease in smallpox cases and advances in laser therapy, not many hospitals today are equipped with this device. In contrast, drills, and burs, which operate under a similar principle, are widely used in plastic surgery and are commonly available in hospitals. Generally, when cutting bone, a carbide bur is preferred and a diamond bur is mainly used to smooth the rough surface of the bone (Fig. 4). We can perform surgery at no additional cost by using an ordinary bur which we already possess.

Difficult hemostasis and severe scar contracture may develop if the entire dermal layer is injured by inappropriate depth control $[3,5,6]$. It is important to preserve not only the cartilage framework but also the pilosebaceous appendages to allow reepithelialization. We therefore set the speed of our drill to its maximum of 30,000 rpm for precise depth control, and used a small round diamond bur for the delicate contouring of the nasal aesthetic unit.

However, in this case, we were able to control bleeding by injecting a vasoconstrictor prior to excision and using hyaluronidase, which promotes the spreading of the locally injected solution. This not only led to more effective hemostasis but also lowered distortion after injection [14]. There have been reports of using the latest instruments to treat rhinophyma. However, along with relevant literature review, we present a case of giant rhinophyma treated effectively at no additional cost using only bone surgery equipment.

\section{NOTES}

\section{Conflict of interest}

No potential conflict of interest relevant to this article was reported. 


\section{Ethical approval}

The study was approved by the Institutional Review Board of Jeju National University Hospital (IRB No. JEJUNUH 2020-03016) and performed in accordance with the principles of the Declaration of Helsinki. Written informed consent was obtained.

\section{Patient consent}

The patient provided written informed consent for the publication and the use of his images.

\section{ORCID}

Jae Kyoung Kang https://orcid.org/0000-0003-3547-6845 Jae Seong Lee https://orcid.org/0000-0002-0087-8791 Jae Hyuck Choi https://orcid.org/0000-0002-7834-7915 Myoung Soo Shin https://orcid.org/0000-0002-8450-821X Byung Min Yun

\section{REFERENCES}

1. Wolter A, Scholz T, Liebau J. Giant rhinophyma: a rare case of total nasal obstruction and restitutio ad integrum. Aesthetic Plast Surg 2017;41:905-9.

2. Karacor-Altuntas Z, Dadaci M, Ince B, Altuntas M. A new surgical technique of rhinophyma (gull-wing technique). J Craniofac Surg 2015;26:e28-30.

3. Sadick H, Goepel B, Bersch C, Goessler U, Hoermann K, Riedel F. Rhinophyma: diagnosis and treatment options for a disfiguring tumor of the nose. Ann Plast Surg 2008;61:114-20.

4. Park CY, Chang H, Jeong EC, Lee YJ. A case of giant rhinophy-

ma showing difficulty in nasal respiration. J Korean Soc Aesthetic Plast Surg 2008;14:165-9.

5. Rohrich RJ, Griffin JR, Adams WP Jr. Rhinophyma: review and update. Plast Reconstr Surg 2002;110:860-9.

6. Bogetti P, Boltri M, Spagnoli G, Dolcet M. Surgical treatment of rhinophyma: a comparison of techniques. Aesthetic Plast Surg 2002;26:57-60.

7. Lutz ME, Otley CC. Rhinophyma and coexisting occult skin cancers. Dermatol Surg 2001;27:201-2.

8. Dufresne RG, Zienowicz RJ, Rozelle A, Whalen JD. An introduction of the ultrasonic scalpel: utility in treatment of rhinophyma. Plast Reconstr Surg 1996;98:160-2.

9. Morandi EM, Verstappen R, Metzler J, Kronberger P, Pierer G, Djedovic G. An old problem with a new solution: cost-effective, easy correction of rhinophyma using a disposable razor. Arch Plast Surg 2018;45:490-2.

10. Ozkan A, Topkara A, Ozcan RH, Senturk S. The use of the PlasmaBlade and acellular dermal matrix in rhinophyma surgery: a case report. J Cutan Med Surg 2016;20:155-8.

11. Taghizadeh R, Mackay SP, Gilbert PM. Treatment of rhinophyma with the Versajet Hydrosurgery System. J Plast Reconstr Aesthet Surg 2008;61:330-3.

12. Alt TH. Facial dermabrasion: advantages of the diamond fraise technique. J Dermatol Surg Oncol 1987;13:618-24.

13. Kim EK, Hovsepian RV, Mathew P, Paul MD. Dermabrasion. Clin Plast Surg 2011;38:391-5.

14. Krausz AE, Goldberg DJ, Ciocon DH, Tinklepaugh AJ. Procedural management of rhinophyma: a comprehensive review. J Cosmet Dermatol 2018;17:960-7. 